\title{
Réalisation de guides d'ondes segmentés monomodes sur une grande plage de longueurs d'ondes réalisés par échange d'ions $\mathbf{A g}^{+} / \mathbf{N a}^{+}$
}

\author{
Davide Bucci, Jérôme Grelin, Elise Ghibaudo, Jean-Emmanuel Broquin
}

Institut de Microélectronique, Electromagnetisme et Photonique 23, rue des Martyrs, 38016 Grenoble CEDEX 1

\begin{abstract}
Dans cet article, on rapporte la réalisation de guides d'ondes segmentés enterrés réalisés en optique intégrée sur verre. Avec la technique de l'échange d'ions, on a pu obtenir des guides monomodes sur les trois fenêtres des télécommunications optiques, de bonne qualité, avec des périodes de segmentation compris entre $3 \mu \mathrm{m}$ et $100 \mu \mathrm{m}$ et des taux de vide compris entre 0,25 et 0,90 . Les effets de la segmentation sont évidents dans la taille modale et dans le déplacement de la longueur d'onde de coupure du deuxième mode confiné dans le guide.
\end{abstract}

Index Terms-Guides segmentées, optique intégrée sur verre, échange d'ions $\mathrm{Ag}^{+} / \mathrm{Na}^{+}$, enterrage, modicité, taille modale

\section{INTRODUCTION}

Durant ces dernières années la réalisation de circuits intégrés optiques a connu un développement considérable surtout en vue des applications liées aux télécommunications optiques. La réalisation de guides segmentés a soulevé un certain intérêt pour les applications de génération de deuxième harmonique dans des matériaux comme le KTP et le niobate de lithium[1]; dans ces cas, l'inversion de domaine et le guidage sont obtenus dans le même temps par diffusion de $\mathrm{Rb}$ et de Ti.

Un guide segmenté est réalisé avec un guide d'onde diélectrique qui est périodiquement coupé par une zone non guidante (voir fig. 1) sur des segments qui sont beaucoup plus longs que la longueur d'onde du signal qui s'y propage. Le rapport entre la longueur de la zone non guidante et la periode des segments est appelé taux de vide. Meme s'il y a eu un grand nombre d'études théoriques sur les guides segmentés, à notre connaissance, la réalisation expérimentale de ce dispositif n'a pas encore été décrite dans le cadre des composants réalisés par échange d'ions sur verre et on se propose donc ici de traiter cette technologie. L'avantage, dans ce cas, est la possibilité de faire varier facilement l'indice effectif des modes guidés et donc de modifier des paramètres comme la plage de monomodicité ou le confinement des modes, en modifiant les dimensions des segments. On gagne donc un degré de liberté en plus sur les caractéristiques de propagation du champ sans pourtant ajouter des étapes technologiques supplémentaires. Dans un premier moment, on concentrera notre attention sur les aspects de réalisation de guides enterrés pour ensuite passer à la description des résultats de mesure, en particulier la taille du mode fondamental et l'analyse spectrale des composants obtenus.

\section{RÉAlis ATION DE GUIDES SEGMENTÉS AVEC L'ÉCHANGE D'IONS}

L'échange d'ions est une technique qui permet de modifier localement l'indice de réfraction d'un verre en faisant varier sa

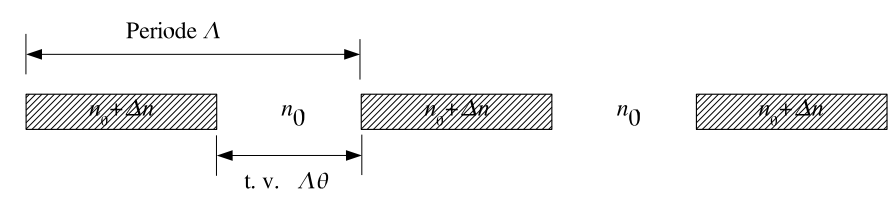

Fig. 1. Sch'ema de principe d'un guide segment'e

composition[2]. La mise en oeuvre consiste à plonger la plaquette dans un sel fondu contenant des ions $\mathrm{Ag}^{+}$qui peuvent diffuser dans le verre en se substituant aux ions $\mathrm{Na}^{+}$qui y sont normalement présents. L'utilisation de la photolithographie permet la réalisation de guides confinés. Il faut néanmoins tenir en compte qu'il s'agit d'un processus de diffusion isotrope et les ions tendent à diffuser longitudinalement sous le masque en réduisant la distance entre les segments. Dans un deuxième temps, on peut appliquer un champ électrique pour faire migrer les ions $\mathrm{Ag}^{+}$vers l'intérieur du verre et réaliser des guides enterrés.

Pour nos essais, on a utilisé un verre silicate spécialement conçu pour l'échange d'ions $\mathrm{Ag}^{+} / \mathrm{Na}^{+}$et on a préparé un échantillon avec un masque comportant plusieurs séries de guides segmentés de $2 \mu \mathrm{m}$ de largeur. Chaque série était composée par des guides segmentés avec un taux de vide variable entre 0,25 et 0,9 et une période fixée. On avait à disposition les périodes de $3 \mu \mathrm{m}, 10 \mu \mathrm{m}, 50 \mu \mathrm{m}$ et $100 \mu \mathrm{m}$. Pour réaliser des guides enterrés, on a effectué un premier échange d'ions dans un mélange de $\mathrm{AgNO}_{3} 20 \%$ molaire et de $\mathrm{NaNO}_{3} 80 \%$ molaire fondu, maintenu à une température constante de $330^{\circ} \mathrm{C}$ pendant $4 \mathrm{~min}$. Après retrait du masque, on a effectué l'enterrage à $10 \mu \mathrm{m}$ en utilisant un bain avec un eutectique contenant du sodium à $260^{\circ} \mathrm{C}$ pendant $1 \mathrm{~h} 30$ avec un courant de $20 \mathrm{~mA}$.

Dans un guide segmenté on a deux paramètres importants (fig. 1): le premier est la période des segments et le deuxième est le taux de vide. Des modèles théoriques[3] ont montré qu'il y a la possibilité de considérer des conditions de propagation qui sont tout à fait similaires à celles d'un guide d'ondes non segmenté caractérisé par une différence d'indice plus faible avec le substrat. Une modification du taux de vide permet donc de faire varier l'indice de réfraction moyen et la période de la structure a une influence très importante vis à vis des pertes par propagation. Les caractérisations seront donc menées de façon à mettre en évidence ces comportements.

\section{CARACTÉRISATION}

La première mesure effectuée a été la taille du mode guidé à $1550 \mathrm{~nm}$ avec un banc de champ proche: la lumière en sortie 


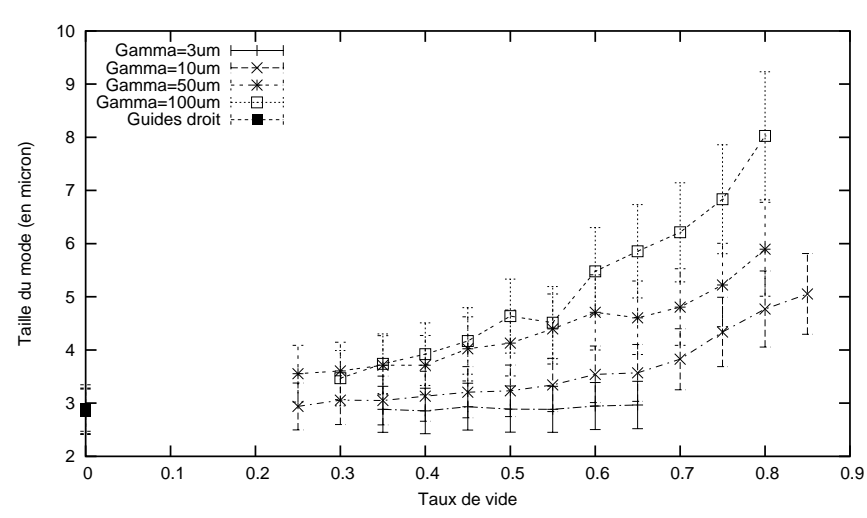

Fig. 2. Tailles du mode fondamental `a $1 / 2$ mesur’e sur un banc de champ proche `a $1550 \mathrm{~nm}$.

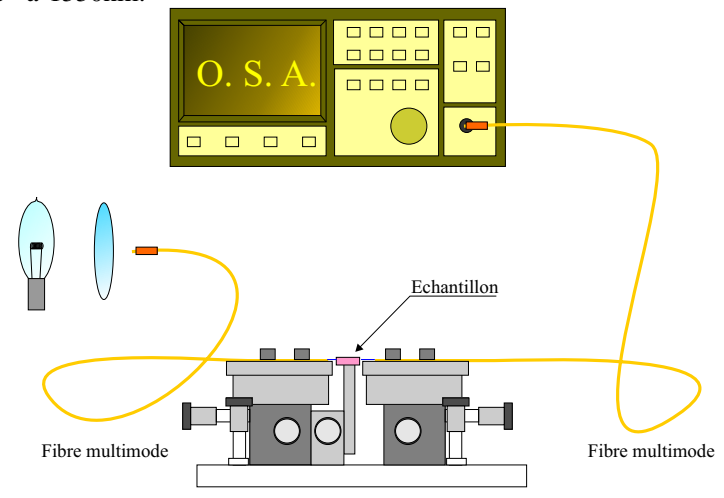

Fig. 3. Fonctionnement du banc d'analyse spectrale pour la caract'erisation modale des guides.

du guide est focalisée sur une caméra CCD InGaAs avec un objectif de microscope. Si on suppose que le profil d'intensité du champ en sortie est approximativement gaussien, la variation de son diamètre à $1 / \mathrm{e}^{2}$ donne une idée du confinement du mode fondamental dans le guide, paramètre qui est étroitement lié à l'indice effectif du mode. Ces mesures (fig. 2) ont été effectuées après un étalonnage pour connaître les tailles en micron; la précision reste néanmoins de l'ordre de $15 \%$. Les résultats sont montrés en fig. 2: on note bien qu'il y a une variation très évidente de la taille modale selon le taux de vide. On remarque en particulier que la variation est plus importante pour les périodes plus grandes $(50 \mu \mathrm{m}$ et $100 \mu \mathrm{m})$. Ce fait peut être expliqué grace à la diffusion des ions qui fait diminuer le taux de vide par rapport au masque, cette différence étant plus marquée pour les périodes courtes.

Les effets de la segmentation sont évidents aussi dans la modicité des guides. Une méthode d'analyse classique consiste à envoyer de la lumière blanche par une fibre multimode à l'entrée du guide à caractériser (fig. 3). La transmission du guide d'ondes sera fortement dépendant des modes guidés et, en récupérant la lumière en sortie avec un analyseur de spectre, on peut observer des marches correspondantes aux coupures des modes (fig. 4). On a pu appliquer cette technique aux guides de période $3 \mu \mathrm{m}, 10 \mu \mathrm{m}, 50 \mu \mathrm{m}$ et les résultats sont visibles en fig. 5. En augmentant le taux de vide, on observe que on peut décaler la longueur d'onde de coupure du deuxième mode, phénomène qui s'accorde bien avec la

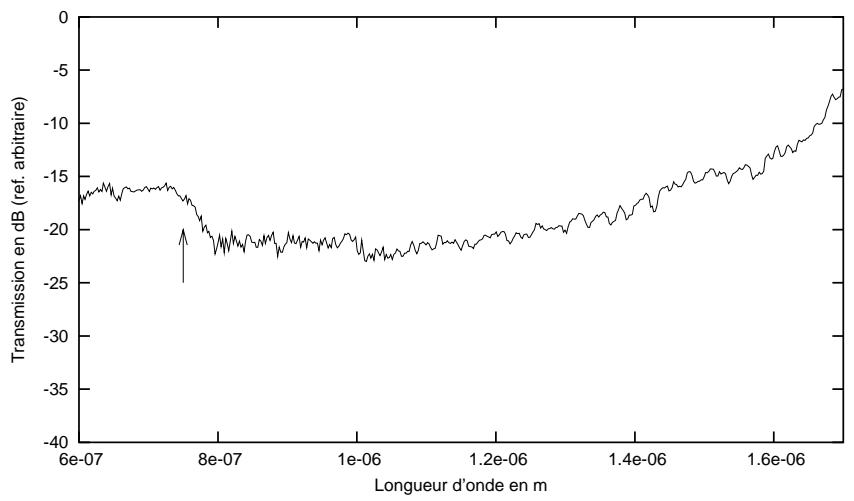

Fig. 4. Analyse spectrale sur un guide de p'eriode $10 \mu \mathrm{m}$ et de taux de vide 0,75 . La fl`eche indique la coupure du deuxi`eme mode; celle du mode fondamental est sup'erieure ‘a 1700nm

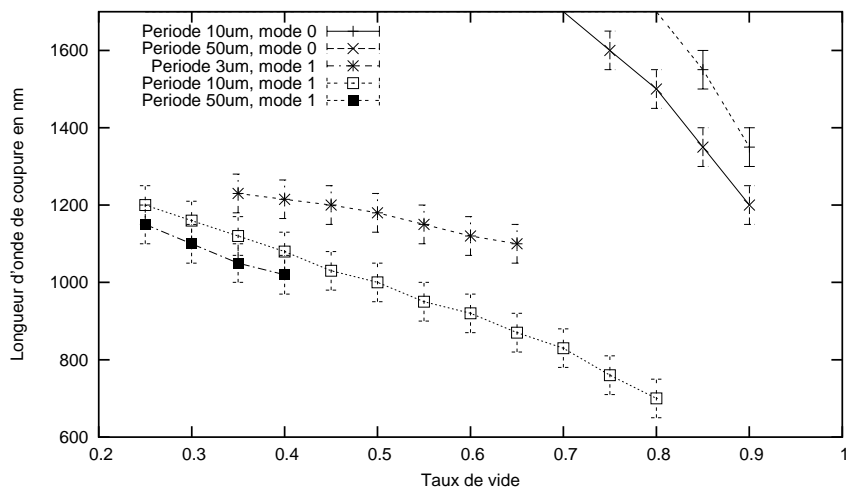

Fig. 5. Longueurs d'ondes de coupure par rapport au taux de vide pour les diff'erentes p'eriodes.

théorie. On remarque en particulier la courbe correspondante à la période de $10 \mu \mathrm{m}$ avec un taux de vide de 0,75 qui est monomode entre $800 \mathrm{~nm}$ et $1700 \mathrm{~nm}$ (fig. 4). De plus, on a relevé la puissance en sortie de chaque guide segmenté en la normalisant avec la moyenne de celle obtenue en sortie des guides droits. Cette mesure a montré que pour les périodes de $10 \mu \mathrm{m}$ on a $(0,7 \pm 0,2) \mathrm{dB} / \mathrm{cm}$ de pertes par propagation en excès. Les guides de période de $100 \mu \mathrm{m}$ n'ont pas pu être caractérisés par analyse spectrale et en fait les pertes en excés deviennent trop importantes, de l'ordre de $(15 \pm 2) \mathrm{dB} / \mathrm{cm}$. Toujours sous cet aspect, les guides de période de $3 \mu \mathrm{m}$ ne présentent pas de différences avec les guides droits (au moins à $0,2 \mathrm{~dB} / \mathrm{cm}$ près) tandis que pour les periodes de $50 \mu \mathrm{m}$ les valeurs varient avec le taux de vide entre $(3,6 \pm 0,2) \mathrm{dB} / \mathrm{cm}$ et $(6 \pm 0,2) \mathrm{dB} / \mathrm{cm}$. Ces valeurs sont intéressantes parce qu'elles montrent que pour certaines périodes les pertes peuvent être très faibles, tout en conservant les effets dus à la variation du taux de vide.

\section{CONCLUSION}

Notre travail a démontré la faisabilité de guides segmentés enterrés avec l'échange d'ions sur verre. Les guides réalisés ont des taux de vide compris entre 0,25 et 0,90 avec des périodes différentes et ont été caractérisés de façon à mesurer les tailles modales et les plages de monomodicité. Avec l'utilisation des guides segmentés, il a été possible 
d'obtenir facilement un guide monomode sur une large plage de longueurs d'ondes $(800 \mathrm{~nm}-1700 \mathrm{~nm})$ en utilisant un processus standard d'échange d'ions. Les résultats s'accordent qualitativement avec le modèle théorique qui consiste à considérer une équivalence avec un guide non segmenté ayant une différence d'indice plus faible avec le substrat. La variation du taux de vide permet de faire varier l'indice effectif des modes, alors que la valeur de la période influe sur les pertes par propagation. Des travaux sont en cours pour étudier plus en détail ce dernier aspect.

Les guides segmentés mettent à disposition une possibilité en plus pour jouer sur les caractéristiques de propagation du champ en faisant varier des paramètres géométriques dans le masque utilisé pour la photolithographie. Cela permet d'éviter de processus plus complexes comme le double échange d'ions et ouvre des possibilités très intéressantes pour des développements futurs.

\section{REFERENCES}

[1] D. Nir, Z. Weissman, S. Ruschin, A. Hardy. Periodically segmented waveguides in Ti:LiNbO 3 . Optics Letters, vol. 19, no. 21, Nov. 1, 1994

[2] J.-E. Broquin. Ion Exchanged Integrated Devices. Integrated Optics Devices V, G. C. Righini, S. Honkanen, Editors, Proc. of SPIE Vol. 4277 pag. 105-117, 2001.

[3] Z. Weissman, A. Hardy. Modes of Periodically Segmented Waveguides. Journal of lightwave technology, vol. 11, no. 11, Nov. 1993. 